\title{
Energy Saving Algorithm of Zigbee Network based on Whole Network Dormancy
}

\author{
Zhi Ren, Yanan Zhao, Hongwei Cao \\ Chongiqng Key Lab of Mobile Communications Technology, Chongqing University of Posts and \\ Telecommunications, Chongqing 400065, China
}

Keywords: Wireless sensor network, Dormancy, ZigBee technology.

\begin{abstract}
Wireless sensor nodes are usually powered by batteries and then work for a long time after deployment, which means that the energy of nodes could become a bottleneck of network operation. To solve the problem of energy limitation of nodes in wireless sensor network, this paper proposes an energy saving routing algorithm based on whole network dormancy. The algorithm can turn all nodes, including terminal nodes, routing nodes and sink nodes, to sleep, saving energy for each node in the network. At the same time, it designs two sleep strategies, named long sleep and short sleep strategy, for routing nodes. The two strategies can further reduce consumption under the premise of not affecting energy consumption. The experimental results based on CC2630 show that: compared with exiting related algorithm, the new algorithm reduces consumption of nodes by $10 \%$.
\end{abstract}

\section{Introduction}

ZigBee is an emerging short-distance and low-rate wireless personal area network technology, which is mainly applied for data transmission and typical periodic communication data transmission among various electronic devices with low communication data size, relatively low data transmission rate and relatively small distribution range [1]. ZigBee technology is developed constantly, which is applied in wireless sensor networks more and more widely with broad application prospects [2]. Wireless sensor network is used in different fields, such as environmental monitoring, industrial system, construction, infrastructure monitoring, etc. [3]. The most important research hotspot is efficient use of energy in wireless sensor networks. Therefore, sensor nodes can enter into dormancy modes for reducing energy consumption and prolonging the service life of networks. It is regarded as the most effective solution [4].

People have put forward many node dormancy algorithms which can be used in the wireless sensor network till present. Existing node dormancy algorithms of wireless sensor networks can be divided into two major categories of synchronous dormancy algorithms and asynchronous dormancy algorithms according to whether the nodes should keep clock synchronization or not. It is required in the synchronous dormancy algorithm that all or a part of nodes of the network keep consistent in clocks (time essentially) (namely the same number or difference fixed value), and the asynchronous dormancy algorithm dos not have synchronization requirements on time node clocks.

In recent years, some asynchronous dormancy algorithms of wireless sensor networks have been proposed successively. An agreement based on lead codes is proposed in the literature [5-8]. However, lead code transmission takes wireless medium for long period of time, thereby reducing throughput and increasing time delay. It is proposed in literature [9] that the receiver starts a duty cycle MAC protocol. Sending nodes send data to target nodes after the sending nodes receive beacon frames sent from the target nodes. Otherwise, the sending nodes should wait. Idle listening time is longer. A node dormancy algorithm in the wireless sensor network is proposed in literature [10], wherein data sending and receiving is considered. Synchronization among all nodes is not required in the algorithm. The dormancy of each node is periodic and independent. When data should be sent by one node, it can be always awake, and data can be sent until the beacon frame from the node is received. The dormancy law of the receiving node can be estimated according to time difference in each beacon (time difference between node wakeup time and beacon sending time). It is not necessary to always wait under waked state when data should be sent next time. Data can be sent directly when the node is awake. An asynchronous dormancy method of sensor nodes based on electrical quantities detection is proposed in 
literature [11]. Node dormancy is arranged in the method according to the predetermined dormancy period. In addition, the electrical quantities of sensor node batteries can be detected in real time. The dormancy time of sensor nodes can be prolonged if the electrical quantities are lower than the set threshold, thereby saving energy consumption. Energy of low electrical quantities nodes is protected. In the method, energy protection of the low electrical quantities nodes is considered. However, the coordination between data transmission and dormancy in actual circumstances is not considered. An algorithm considering the dormancy of wireless sensor nodes in the working period is proposed in literature [12]. The wireless sensor node is capable of double dormancy by the algorithm. However, the adverse influence of listening cycles on energy-saving effects is fixed in the algorithm. A node dormancy awakening strategy based on the ZigBee technology is designed in Literature [13]. Routing nodes always keep the working state by the strategy; sensor nodes without routing tasks (hereinafter referred to as 'terminal nodes') can undergo periodic dormancy. Dormancy of routing nodes in idle state is not considered.

Insufficient and inaccurate design is considered by the above-mentioned dormancy algorithm in the aspect of dormancy strategies in different logical type node. In addition, the mode of fixing listening cycle length within the working period may affect the energy saving effect. Therefore, an energy saving algorithm of routing nodes and terminal nodes based on dormancy is proposed in the paper. The routing nodes and the terminal nodes are set in dormancy. In addition, different dormancy strategies are adopted. Routing nodes are divided into working periods and dormancy periods. In addition, short dormancy is added in the working period under the precondition of not affecting data forwarding in routing nodes. The routing nodes can adapt to dormancy in the working period, thereby reducing energy consumption of the routing nodes.

\section{ZigBee Network Topological Structure}

A tree cluster network topological structure is adopted in the plan. A PAN network is established by coordinator nodes in figure 1. Routing node: it plays the role of data forwarding; terminal node: it is mainly responsible for periodic data acquisition and transmission to remote terminals. The coordinator nodes are initiators and managers to establish the ZigBee network [14]; they are not involved in dormancy. Routing nodes and terminal nodes are powered by batteries, which can undergo periodic dormancy and awakening, thereby saving energy and prolonging the service life the network.

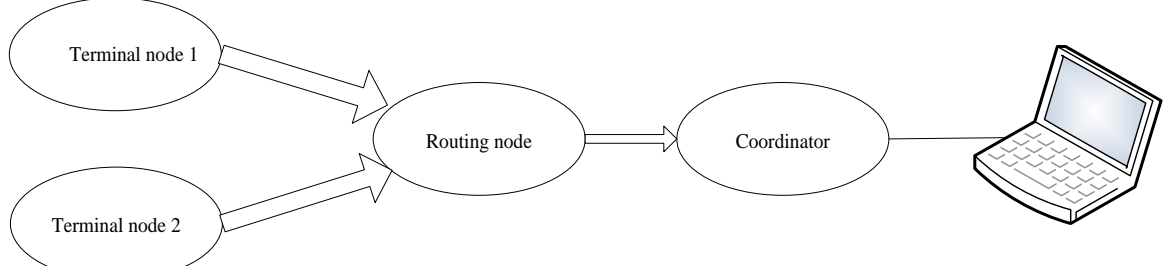

Fig. 1 ZigBee Network Topological Structure

\section{Energy-saving Routing Algorithm of ZigBee Network}

\subsection{Energy Saving Algorithm of Routing Node Based on Dormancy}

Working time of nodes is divided into a dormancy period and a working period with equal length by the algorithm in the paper. Dormancy is also available under the precondition of guaranteeing that all data sent from the terminal nodes are received if tasks are not received and sent by the routing nodes within the working period. Namely, there are many dormancy times within one cycle period. In the paper, the dormancy period is set as $2 \mathrm{~s}$, and the working period is set as $1 \mathrm{~s}$. There are many short dormancy cycles within the working period. Short dormancy period is 960us. The characteristic of the node is fully utilized during work for $1 \mathrm{~s}$, namely each conflict should be prolonged randomly. Nodes are set in dormancy as far as possible under the condition of guaranteeing that all data can be received. CSMA/CA mechanism is adopted by nodes in the network for data transmission. The default retransmission frequency is 3 . The retreating time after node conflicts is calculated as follows: one 
integer value is selected randomly in [0, 2BE-1], which is multiplied by unit retreating time ( $20 * 16$ us $=320 \mathrm{us}$ ), wherein the minimum $\mathrm{BE}$ [15] is 3 , the maximum value is 5,1 is added after reach retransmission, but the value should not be more than 5 .

There are a total of 5 states of routing nodes in the whole dormancy period: listening state, data forwarding state, short dormancy state, long dormancy state and startup state. Concrete operation of each state is shown as follows:

Listening state: the routing node monitors whether some nodes desire to send data to the routing node or not. The routing node forwards data to the sending and receiving state if some nodes send data to the routing node. The listening state lasts for $9.921 \mathrm{~ms}$. The node can directly enter short dormancy if no data is received during the period.

Data sending and receiving state: the routing node under the state is responsible for data sending and receiving, and most energy is consumed.

Short dormancy state: The time from short dormancy state to normal sending and receiving state is not long compared with long dormancy, about 400us. States can be converted flexibly.

Long dormancy state: a few energy of routing nodes can be consumed under the state. Energy can be saved maximally if the node is set under the state for long time. However, long time should be spent by the routing node from long dormancy state to normal data state, about $10 \mathrm{~ms}$ (obtained through experimental test).

Startup state: it refers that the routing node lasts from short dormancy stage to normal data sending and receiving stage.

The state transition of the routing nodes is shown in figure 2:

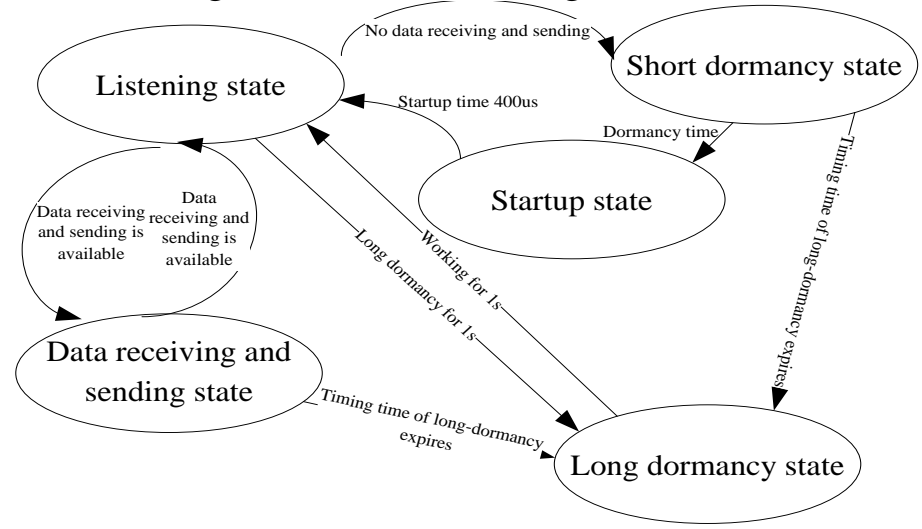

Fig. 2 Data Transition Drawing

Concrete energy saving algorithm is shown as follows:

1) Firstly, the routing RA node is set in the listening state, and listening lasts for $9.921 \mathrm{~ms}$ during the working period of the routing node. The node can enter data receiving and sending stage and data is received and sent for tDATA time if the node RA receives any data at any time during the period. Data sending and receiving are finished, and the nodes enter the listening state after tDATA time. It is proved that no nodes send data to the routing node RA if no data packet is received by the routing node RA within the routing time for $9.921 \mathrm{~ms}$, and the routing node will enter the short dormancy state.

2) The routing node is awakened automatically after short dormancy for 560us. 400us is consumed by the routing node for converting from the dormancy state to normal state of data sending and receiving. Listening stage is continued in the node after the 400us. The routing node enters the data receiving and sending stage if data sending is monitored, thereby indicating that data receiving and sending are finished.

3) The routing node can directly enter the dormancy state after $n$ periods if the time since the first listening to present is $1 \mathrm{~s}$. The routing node enters the first listening state of $1 \mathrm{~s}$ working stage again after long dormancy for $1 \mathrm{~s}$, and the operation is repeated periodically.

Routing node work sequence chart is shown in figure 3: 


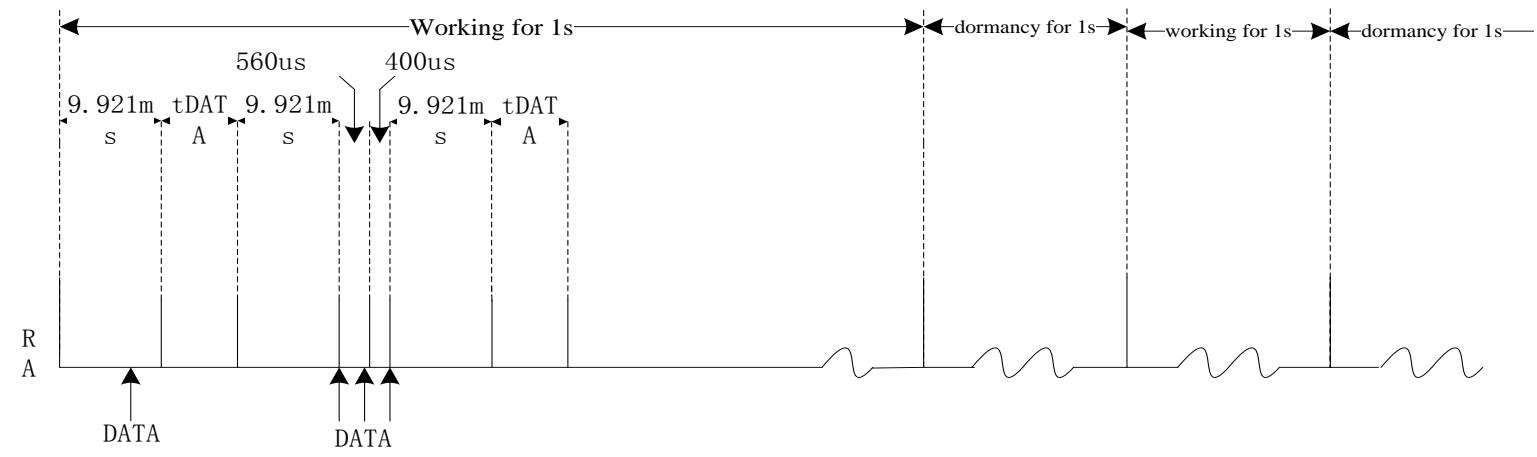

Fig. 3 Routing node work sequence

Basis and meaning of all time settings are shown as follows:

Dormancy period: it is set as $2 \mathrm{~s}$, working time is set as $1 \mathrm{~s}$, and dormancy time is set as $1 \mathrm{~s}$.

Listening time: it is set as $9.921 \mathrm{~ms}$. it refers to the time that the maximum retreating time window of data retransmission plus data transmission time after node conflicts.

Maximum retreating time window: it is set as $9.92 \mathrm{~ms}$. since one number is randomly selected in [0, 2BE-1], which is multiplied by $20 * 160$ as the retreating time, the maximum retreating time is $31 * 20 * 16=9.92 \mathrm{~ms}$.

Message transmission time: it is assumed that the transmission distance is $300 \mathrm{~m}$, the speed of light is $299792458 \mathrm{~m} / \mathrm{s}$, so the transmission time is $300 \mathrm{~m} /(299792458 \mathrm{~m} / \mathrm{s})=1$ us.

Data sending and receiving time (tDATA): it refers to the time since the node starts to receive and send data packets until it stops receiving and sending data packets.

Short dormancy time: it is set as 560us. Data retransmission frequency limit is 3 . It is believed that data transmission is failed if data is still not transmitted successfully after data retransmission for three times. It is assumed that the routing node just enters dormancy when the node B retransmits data for the first time. Since the retreating time unit is $320 \mathrm{us}$, the routing nodes can normally receive and send data for at most $3 * 320 \mathrm{us}=960 \mathrm{us}$ in order to ensure that data can be sent by the routing node under normal work state in at least one of three data transmissions, wherein 400us is the time consumed by the routing node from dormancy state to normal time of sending and receiving data. Therefore additional 960us-400us=560us is regarded as short dormancy time of the routing node.

Startup time: it is set as 400 us. It refers to the time consumed by the node from the router state to normal data receiving state.

\subsection{Realization of Terminal Node Energy Saving Algorithm Based on Dormancy}

The terminal node is responsible for periodic data collection. Data is uploaded to the coordinator through the routing node. The timing time depends on whether data is sent or not in the terminal node through a timer. The terminal node is awakened, the time stamp 1 under the circumstance is recorded. The system can check whether data should be sent or not in the data buffer zone. Data can be sent if it is available until the last data is sent. The time stamp 2 under the circumstance is recorded. The terminal node enters the dormancy state after data sending. Dormancy time is stamp $=1-(\operatorname{stamp} 2-\operatorname{stamp} 1)$. Dormancy is $1 \mathrm{~s}$ if no data is sent. The above process is implemented continuously after the node is awakened.

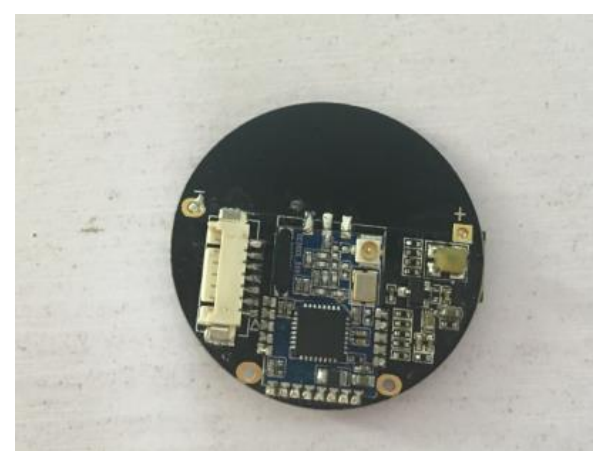

Fig. 4 Algorithm Verification Test on ZigBee Node 


\subsection{Algorithm Verification}

The algorithm is verified on a CC2630 chip. The node belongs to a fully-functional node. Different codes are downloaded, and different functions are implemented. The hardware structure is shown in figure 4 . The lithium battery for test is about $200 \mathrm{~mA} \cdot \mathrm{h}$.

The node topological structure is shown in figure 5 in the algorithm verification.

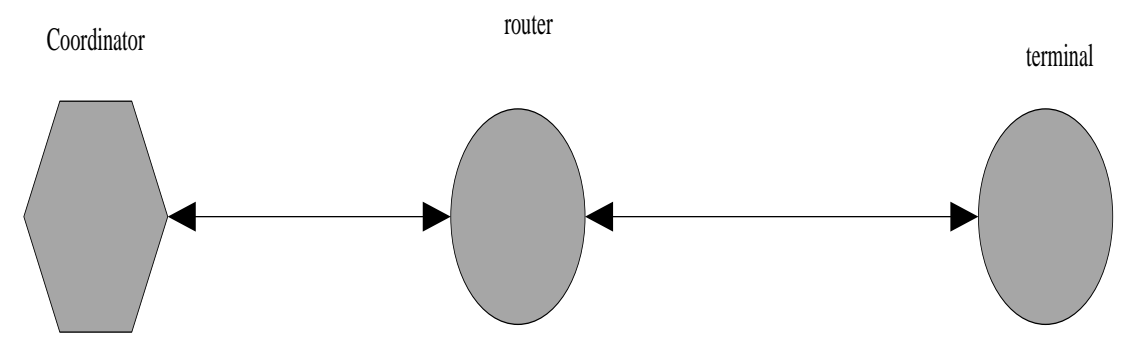

Fig. 5 ZigBee Network Structure of Dormancy Algorithm Verification

Wherein, the test is implemented indoors. The distance among the coordinator, the router and the endpoint is $5 \mathrm{~m}$ respectively. The energy saving algorithm is operated in the router and the terminal after all nodes are electrified and networked successfully. The dormancy time is $1 \mathrm{~s}$, and the working time is $1 \mathrm{~s}$ during test.

The current of the CC2630 chip is tested by an instrument in real time. The current during normal work is $6.71 \mathrm{~mA}$. The dormancy current is $139.11 \mathrm{uA}$. The energy saving algorithm test results of the routing node and the terminal node based on dormancy proposed in the paper are respectively shown in figure 6 and figure 7.

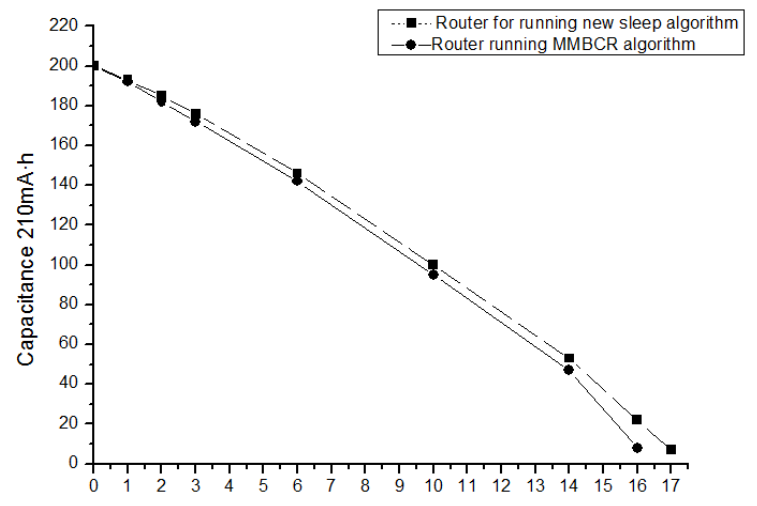

Fig. 6 Routing node energy saving algorithm verification based on dormancy

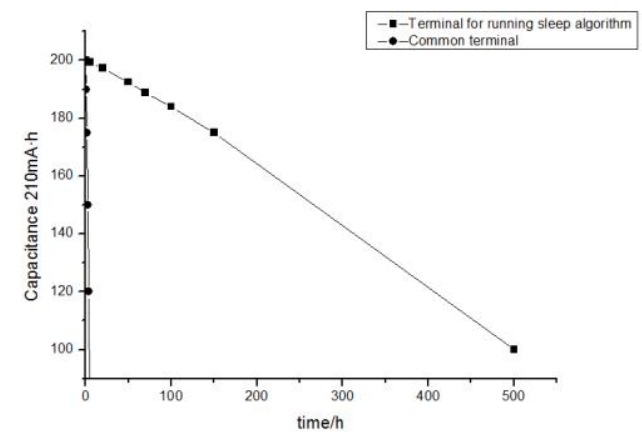

Fig. 7 Terminal node energy saving algorithm verification based on dormancy

The feasibility of applying MMBCR (Min-Max Battery Cost Routing) algorithm in ZigBee network is proposed and verified through simulation in literature [16-17]. The algorithm is simulated on the CC2630 platform, and compared with new algorithms. Figure 6 shows that the battery has the same electric capacity; meanwhile power is supplied to the router with operation of new dormancy algorithm and the router with operation of MMBCR algorithm. The electric capacity shows that the new algorithm 
can save $10 \%$ of energy with time progression. Figure 7 shows that the terminal with operation of dormancy algorithm consumes 50\% of electric capacity of the test battery after being used for 500 times when common terminal router consumes all electric capacity of the test battery for $7 \mathrm{~h}$. Algorithm validation shows that the energy saving algorithm of the routing node and terminal node based on the dormancy can reduce the power consumption of the router and the terminal effectively, thereby reaching the purpose of saving energy in the network.

\section{Summary}

In the paper, an energy saving algorithm of the routing node and terminal node based on the dormancy is proposed through research on MAC protocols and the related algorithms aiming at the problem that node energy is limited in the wireless sensor network. The two energy saving algorithms are verified on the CC2630 platform. Experimental results show that the algorithm proposed in the paper can reduce the energy consumption of the routing node and the terminal node effectively, thereby prolonging the service life of the network. Key parameters of new algorithm can be further optimized in future work.

\section{References}

[1] Gao Xiang, Deng Yongli, Lu Yuanyuan, etc. Research on ZigBee network energy saving algorithm based on Z - Stack protocol stack. Chinese Journal of Structural Chemistry, 2014, 27 (11) : 1534-1538.

[2] Guo Yuanbo, Yang Kuiwu, Zhao Jian, etc. Application of ZigBee technology. Beijing: National Defence Industry Press, 2010.

[3] Sun Limin, Yan Tingshen, Bi Yanzhong. Wireless sensor network and application. Beijing: Tsinghua University Press, 2005.

[4] Azevedo J,Santos F, Rodringues M,et al. Sleeping ZigBee networks at the application layer. Iet Wireless Sensor Systems, 2014, 4(1): 35-41.

[5] Polastre J,Hill J,Culler D. Versatile low power media access for wireless sensor networks. International Conference on Embedded Networked Sensor Systems. New York, USA: ACM, 2004:95-107.

[6] Buettner M,Tee G V,Anderson E,et al. X-MAC: a short preamble MAC protocol for duty-cycled wireless sensor networks. Proceeding of the 4th international conference on Embedded networked sensor systems. New York, USA: ACM, 2006:307-320.

[7] EL-Hoiydi,Decotigmic J D.WiseMAC:An UltraLow Power MAC Protocol for Multi-hop Wireless Sensor Networks. Algorithmic Aspects of Wireless Sensor Networks:First International Workshop. Turku, Finland: Proceedings, 2004:18-31.

[8] Sun Limin, Yan Tingshen, Bi Yanzhong, etc. Listening dormancy method of wireless sensor network MAC layer. Chinese Patent; Application Number CN200610012187.2, 2007. 12.

[9] Sun Y,Gurewitz O,Johnson D B. RI-MAC:a receiver-intiated asynchronous duty cycle MAC protocol for dymac traffic loads in wireless sensor networks. International Conference on Embedded Networked sensor Systems. New York, USA: ACM, 2008:1-14.

[10] Lee H,Hong J,Yang J,et al.A pseudo-Random Asynchronous Duty cycle MAC Protocol in Wireless Sensor Networks.IEEE Communications Letters, 2010, 14(2): 136-138.

[11] Li Mi, Hua Shihai, Li Guike, etc. WSNs intelligent dormancy method based on electric capacity detection. Transducer and Microsystem Technologies, 2014, 33 (11): 27-29.

[12] Xu Ke, Qu Yi, Chen Wenlong. Dual dormancy method of wireless sensor node, Chinese Patent, Application number: CN20130474024.6, 2013.10.

[13] Kong Qingyan, Su Shuzhi. Dormancy awakening trategy based on ZigBee [EB/OL]. [EB/OL]. 2011.8, http://www.21ic.com/app/rf/201108/91354.htm.

[14] Wang Zhifan, Ye Qingwei, Zhou Yu, etc. Low power wireless sensor technology based on queuing theory and application thereof. Computer Engineering, 2016, 42(8): 39-45. 
[15] Wireless Medium Access Control (MAC) and Physical Layer (PHY) Specifications for Low-Rate Wireless Personal Area Networks (LR-WAPNs), IEEE 802.15.4 Standard.oct, 2003.

[16] Tekbiyik N, Uysal-Biyikoglu E. Review: Energy efficient wireless unicast routing alternatives for machine-to-machine networks. Journal of Network \& Computer Applications, 2011, 34(5): 1587-1614.

[17]Zhang Z, Zhou F, Liang Y. ZigBee routing algorithm based on energy awareness and energy balance. International Conference on Consumer Electronics, Communications and Networks. IEEE, 2012:747-750.

[18] Toh C K. Maximum battery life routing to support ubiquitous mobile computing in wireless ad hoc networks. IEEE Communications Magazine, 2001, 39(6):138-147. 